\title{
उMR
}

\section{Characterization and comparison of flower bud microRNAs from yellow-horn species}

\author{
Y. $\mathbf{A 0 ^ { 1 , 2 }}$ \\ ${ }^{1}$ Key Laboratory for Silviculture and Conservation, \\ Ministry of Education, Beijing Forestry University, Beijing, \\ People's Republic of China \\ ${ }^{2}$ National Energy R\&D Center for Non-food Biomass, Beijing, \\ People's Republic of China \\ Corresponding author: Y. Ao \\ E-mail: aoyan316@163.com
}

Genet. Mol. Res. 15 (4): gmr.15048899

Received June 17, 2016

Accepted August 9, 2016

Published October 6, 2016

DOI http://dx.doi.org/10.4238/gmr.15048899

Copyright (C) 2016 The Authors. This is an open-access article distributed under the terms of the Creative Commons Attribution ShareAlike (CC BY-SA) 4.0 License.

ABSTRACT. An increasing number of microRNAs (miRNAs) are
known to play crucial regulatory roles in floral organ differentiation
and development. Yellow-horn (Xanthoceras sorbifolia Bunge) is an
oil-rich seed shrub that has great potential for biodiesel production. To
evaluate the roles of miRNAs in the regulation of flower development,
we used high-throughput sequencing to characterize them from flower
buds of double-flower yellow-horn mutant and wild-type plants.
Approximately 33 million raw reads were obtained through Solexa
sequencing of two small RNA libraries, and the conserved and novel
miRNA locations were annotated. Nineteen conserved miRNAs
(belonging to nine families) and eight novel miRNAs were identified
from the two libraries. Twelve conserved and eight novel miRNA-
star sequences were also discovered, providing additional evidence
for the existence of miRNAs in this species. Thirteen miRNAs were

Genetics and Molecular Research 15 (4): gmr.15048899 
differentially expressed between wild-type and mutant plants. Realtime reverse transcription-polymerase chain reaction analysis revealed the expression patterns of eight microRNAs, which were confirmed by sequencing. The assessed targets of these miRNAs are involved in transcriptional regulation, disease resistance, antioxidative metabolism, nucleobase regulation, and nucleic acid metabolism. This study opens up new avenues for understanding the roles of miRNAs in X. sorbifolia.

Key words: Xanthoceras sorbifolia Bunge; Double-flower mutant; Wild-type; microRNA; qRT-PCR; High-throughput sequencing

\section{INTRODUCTION}

Owing to increasing concerns about energy shortage and climate change, biodiesel has been increasingly attracting the attention of governments, worldwide. Oil-rich seed plants that can be used to produce renewable and environmentally friendly biodiesel have received considerable attention (Freitas et al., 2011; Zhang et al., 2012; Guo et al., 2014; Yao et al., 2013). Yellow-horn (Xanthoceras sorbifolia Bunge), belonging to the Sapindaceae family, is a wild oilseed tree that has high tolerance to cold, drought, infertile soils, and salinity (Shao and Chu, 2008). The seeds accumulate a significant quantity of oil (55-70\%). The oil is recognized as a high-quality raw material for biodiesel production, based on its physiochemical properties (Van Gerpen, 2005; Zhang et al., 2010). However, the species exhibits two distinct floral morphologies having either single-flowers (wild-type, WT) or double-flowers (mutant-type, MT) (Ao, 2010), with only WT capable of producing seeds and oil. In MT, the stamens and pistils are petaloid, and therefore, they do not bear fruits. Whether this morphological distinction is associated with differences at the molecular level remains unknown.

MicroRNAs (miRNAs) are short, noncoding RNAs involved in post-transcriptional regulation of gene expression and are critical for plant growth and development, stress responses, and other diverse biological processes (Wang et al., 2014). Dozens of miRNAs play key roles in the control of floral organ fate, regulation of flowering time, and differentiation of particular cells during flower development; these include miR156, miR169, miR164, miR319, miR159, miR167, and miR172 (Nagpal et al., 2005; Cartolano et al., 2007; Allen et al., 2007; Nag et al., 2009). However, only a limited number of miRNAs involved in flower development have been identified in woody plants, especially in yellow-horn.

To investigate the miRNAs related to morphological differences between WT and MT, and to unravel their involvement in the regulation of gene expression, we performed a comparative analysis of small RNAs in the WT and MT flower buds, screened them for miRNAs potentially involved in yellow-horn flower initiation, and determined their differential expression patterns. Moreover, we analyzed the expression patterns of the corresponding target genes of the miRNAs. Our results revealed the presence of numerous unidentified miRNAs in yellow-horn buds. This study is expected to provide the basis for further studies on yellow-horn miRNA regulation mechanisms and help in delineating a potential functional network of flower initiation-related miRNAs in yellow-horn buds.

Genetics and Molecular Research 15 (4): gmr.15048899 


\section{MATERIAL AND METHODS}

\section{Plant materials}

WT and MT flower buds were obtained from 40-year-old yellow-horn cultivated in Chengde City, Hebei Province, China. Both WT and MT plants were derived from sexual reproduction of WT, and were raised from naturally crossed seedlings. WT plants exhibited single-flowers, whereas MT plants produced double-flowers. Typically, mature flowers of WT feature three bracts, five sepals, five petals, eight stamens, five yellow filamentous appendages, and one pistil (Figure 1). In MT, supernumerary petals are observed in several additional whorls. The double-flower has approximately 17 to 25 petals and petal-like appendages, three bracts, five sepals, and deformed stamens and pistil (Figure 1). Within each type, individuals have the same kind of flowers, and the flower characteristics remain stable every year. Due to the limited number of MT, 10 representative flower buds were collected from three individuals of each type in the end of March. The samples were frozen in liquid nitrogen and stored at $-80^{\circ} \mathrm{C}$
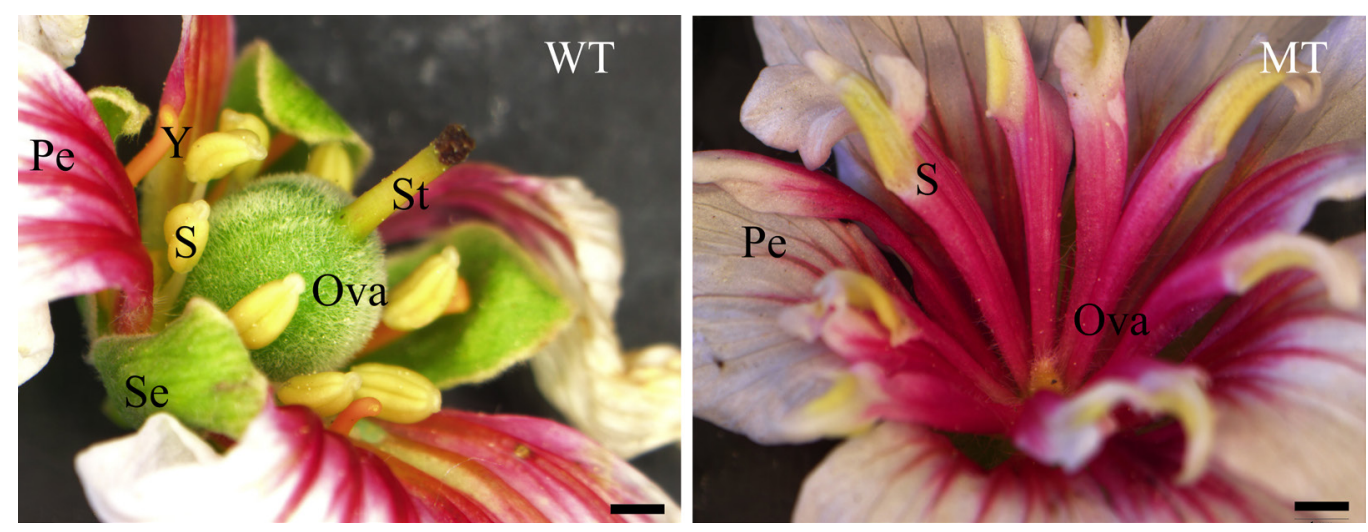

Figure 1. Characteristics of WT and MT yellow-horn flowers. Se, sepal; Pe, petal; S, stamen; Y, yellow filamentous appendages; Ova, ovary; St, style. Bar $=1 \mathrm{~mm}$.

\section{Total RNA isolation, small RNA library construction, and Solexa sequencing}

Total RNAs were extracted from the flower buds of WT and MT plants using Trizol reagent (Invitrogen, Carlsbad, CA, USA). RNA quality was examined on the Agilent 2100 Bioanalyzer (Agilent Technologies, USA). Extracted RNAs were used for small RNA library construction in both the WT and MT groups. miRNA cloning was performed as described previously by Sunkar and Zhu (2004). Small RNAs were sequenced by the Illumina Solexa Genetic Analyzer at the Beijing Genomics Institute (Shenzhen, China).

\section{Bioinformatic analysis of the sequencing data}

Briefly, the adaptor sequences were extracted from the raw reads, and the small RNAs, in turn, were extracted from the adaptors. The length distributions of clean tags were also 
summarized. Unique small RNAs were obtained after eliminating the redundant sequences. Illumina Solexa Hi-seq 2000 high-throughput sequencing technology was used to obtain a comprehensive transcriptome from the flower buds of yellow-horn. A total of 56,632,980 raw reads and 52,869,962 clean reads were obtained. Unique small RNAs were aligned against the yellow-horn transcriptome using Short Oligonucleotide Analysis Package (Li et al., 2008). Small RNA tags matching the mRNA exons and introns were removed. Sequences were aligned to RFam and NCBI GenBank databases to filter rRNAs, tRNAs, snRNAs, snoRNAs, and repeats. To identify the conserved miRNAs, the remaining sequences were analyzed via a BLAST search against miRBase (Release 21), allowing a maximum of two mismatches (Griffiths-Jones et al., 2008). The sequences that could not be annotated were employed to predict novel candidate miRNAs using Mireap (http://sourceforge.net/projects/ mireap/) according to the previously published criteria (Meyers et al., 2008). The miRNA target genes were obtained according to the procedures and criteria described previously (Schwab et al., 2005).

\section{Differential expression analysis of miRNAs}

miRNA expression was compared between the two libraries (WT and MT) to determine the miRNAs that were differentially expressed. The expression was normalized (actual miRNA count/total count of clean reads $x 1,000,000$ ). The normalized reads were analyzed using the Fisher exact test with Bonferroni correction for multiple hypothesis testing. Fold-change was calculated as follows: fold-change $=\log 2$ (normalized reads in the MT library / normalized reads in the WT library). If the fold-change was greater than 1.5 or less than 0.67 , the $\mathrm{P}$ value was less than 0.05 , and the normalized reads in the two libraries were greater than one, the miRNA was considered to be significantly up-regulated or down-regulated, respectively, in the MT flower bud.

\section{Real-time reverse transcription-polymerase chain reaction (qRT-PCR) analysis}

To validate the expression of miRNAs identified by Solexa sequencing, real-time reverse transcription-polymerase chain reaction (qRT-PCR) analysis was performed using BioTeke Super RT Kit PR6601 and 2X SYBR Real-Time PCR Premixture PR7002 in an ABI 7500 Fast Real-time PCR machine (Applied Biosystems, USA) with three replications. The yellow-horn 5.8S rRNA was used as an internal reference. Specific forward primers were designed for each individual miRNA (Table S1) whereas the reverse primer was universal. The reactions were conducted in $20-\mu \mathrm{L}$ volumes containing $1 \mu \mathrm{L}$ diluted cDNA, $0.4 \mu \mathrm{L}$ each primer $(10 \mu \mathrm{M}), 10 \mu \mathrm{L} 2 \mathrm{X}$ TaKaRa qPCR Mix, and $8.2 \mu \mathrm{L} \mathrm{ddH}_{2} \mathrm{O}$. The PCR was performed using the following cycling conditions: $95^{\circ} \mathrm{C}$ for $30 \mathrm{~s}, 45$ cycles of $95^{\circ} \mathrm{C}$ for $5 \mathrm{~s}$ and $65^{\circ} \mathrm{C}$ for $60 \mathrm{~s}$, a thermal denaturing cycle at $95^{\circ} \mathrm{C}$ for $15 \mathrm{~s}, 60^{\circ} \mathrm{C}$ for $30 \mathrm{~s}$, and $95^{\circ} \mathrm{C}$ for $15 \mathrm{~s}$.

\section{Statistical analysis}

miRNA levels in the WT sample were set to 1 . The relative gene expression level was calculated using the comparative $\mathrm{Ct}$ method. $\Delta \mathrm{Ct}$ was calculated based on the formula: $\Delta \mathrm{Ct}=$ $\mathrm{Ct}$ (target miRNA) - Ct (5S rRNA). In addition, $\Delta \Delta \mathrm{Ct}$ was calculated as follows: $\Delta \Delta \mathrm{Ct}=\Delta \mathrm{Ct}$ (experimental group) - $\Delta \mathrm{Ct}$ (control group); $2^{-\Delta \Delta \mathrm{Ct}}$ was taken as the relative expression level.

Genetics and Molecular Research 15 (4): gmr.15048899 


\section{RESULTS}

\section{Small RNA sequence analyses and annotation}

A total of 17,879,296 and 15,842,969 reads were generated from WT and MT flower buds, respectively (Table 1). After removing the adapter sequences and low-quality reads, 17,323,202 (WT) and 15,357,701 (MT) clean reads were obtained. Upon subsequent alignment to RFam and NCBI GenBank databases, 6,730,006 and 5,863,163 unique sequences were obtained from WT and MT flower buds, respectively (Table S2).

\section{Table 1. Summary of small RNA sequences from WT and MT yellow-horn flower buds.}

\begin{tabular}{l|c|c}
\hline MT library (WT library) & Total sRNAs & Unique sRNAs \\
\hline Raw reads & $15,842,969(17,879,296)$ & - \\
\hline Low quality reads removed & $15,604,217(17,599,508)$ & - \\
\hline Clean reads* & $15,357,701(17,323,202)$ & $5,863,163(6,730,006)$ \\
\hline Mapping to the genome & $4,068,291(4,444,343)$ & $469,291(525,614)$ \\
\hline Matching known miRNAs & $1,748,090(1,707,397)$ & $45,370(52,309)$ \\
\hline Unannotated sRNAs & $13,026,379(14,652,365)$ & $5,742,531(6,571,967)$ \\
\hline
\end{tabular}

*Clean reads were those that remained after removing the contaminant reads.

Both WT and MT small RNAs shared a similar distribution pattern, with the highest peak representing the 24-nt size class and a minor peak representing the 22-nt RNAs (Figure 2). The 24-nt RNA class represented 61.81 and $59.54 \%$ of the clean reads from WT and MT libraries, respectively. The 22-nt small RNAs were the second most abundant class, at 13.06 and $14.10 \%$ of the total WT and MT reads, respectively.

\section{a MT $\otimes \mathrm{WT}$}

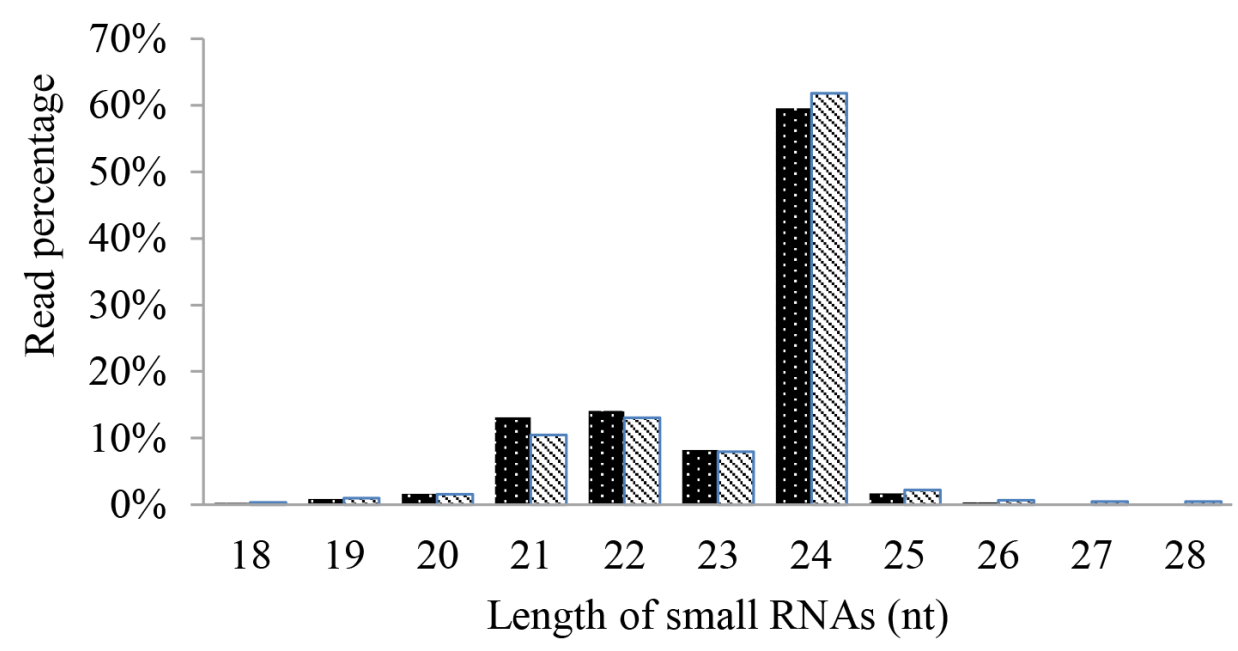

Figure 2. Size distribution of sRNAs in libraries from yellow-horn WT and MT flower buds.

Genetics and Molecular Research 15 (4): gmr.15048899 


\section{Conserved miRNAs expressed in WT and MT flower buds}

A total of 52,309 and 45,370 conserved miRNA sequences were identified from the small RNA libraries of WT and MT flower buds, respectively. The conserved WT and MT miRNAs belonged to eight and nine miRNA families, respectively (Table S3). Among the conserved miRNAs, 19 sequences from nine miRNA families mapped to nucleotide sequences, all of which were capable of adopting hairpin structures resembling the fold-back structure of a miRNA precursor. Twelve miRNA-star sequences (miRNA*) and their complementary strands of functional mature miRNA were also detected (Figure S1).

The expression levels of miRNAs from several families were extraordinarily high in both the libraries (Table S3 $)$. miR3954 was the most abundant, with 930,131 WT and 856,209 MT reads, accounting for 63.60 and $71.88 \%$ of all the conserved miRNA reads, respectively. Most conserved miRNA families were identified in both the libraries, except for miR160d and miR166d, which were exclusively detected in the MT library, and miR171 and miR482b, which were detected in the WT library only. Moreover, the reads of individual miRNAs within a given family varied greatly. For instance, miR166 members in the WT library varied in abundance from 0 to 207,211 reads.

\section{Novel miRNAs identified in WT and MT libraries}

Due to the lack of whole genome sequence data for yellow-horn, novel miRNAs were identified by mapping them to the yellow-horn transcriptome. We identified 33 yellow-hornspecific miRNAs (Table 2). Of these, eight had star sequences and were considered novel miRNAs. The remaining 25 miRNAs were considered potential miRNAs. The hairpins have negative folding free energies. The average minimum free energy value was $-48.08 \mathrm{kcal} /$ mol, with a range of -19.20 to $-67 \mathrm{kcal} / \mathrm{mol}$. More importantly, the identification of eight miRNA* sequences (Rn1, Rn2, Rn3, Rn4, Rn5, Rn6, Rn7, and Rn8) from the candidate novel miRNAs provided more evidence that this classification was appropriate. The structures of the precursors of both the novel and potential miRNAs are presented in Figure S2. Of the 33 yellow-horn-specific miRNAs, 15 miRNAs were found exclusively in MT flower buds, and four were found exclusively in WT.

\section{Differential miRNA expression profiles between WT and MT flower buds}

After the normalization of miRNA reads, we observed that the expression levels of 13 miRNAs (eight conserved and five novel) were significantly different between the WT and MT samples (Table 3). Of these miRNAs, the expression of 11 miRNAs (six conserved and five novel) was at least 1.5-fold higher in the MT buds compared to the WT flower buds, suggesting that these miRNAs were likely the positive regulators of flower-initiation process. Expression of Rn8 in MT flower buds was approximately 5 -fold higher than that in WT flower buds. In contrast, miR160b and miR160c were down-regulated in the MT samples. Suppression of these miRNAs in the mutant suggests that they most likely play negative regulatory roles in the process. Overall, we observed considerable variability between the two variants of yellowhorn with regard to the abundance of miRNA families.

Genetics and Molecular Research 15 (4): gmr.15048899 
Table 2. Yellow-horn-specific miRNAs identified in the WT and MT libraries.

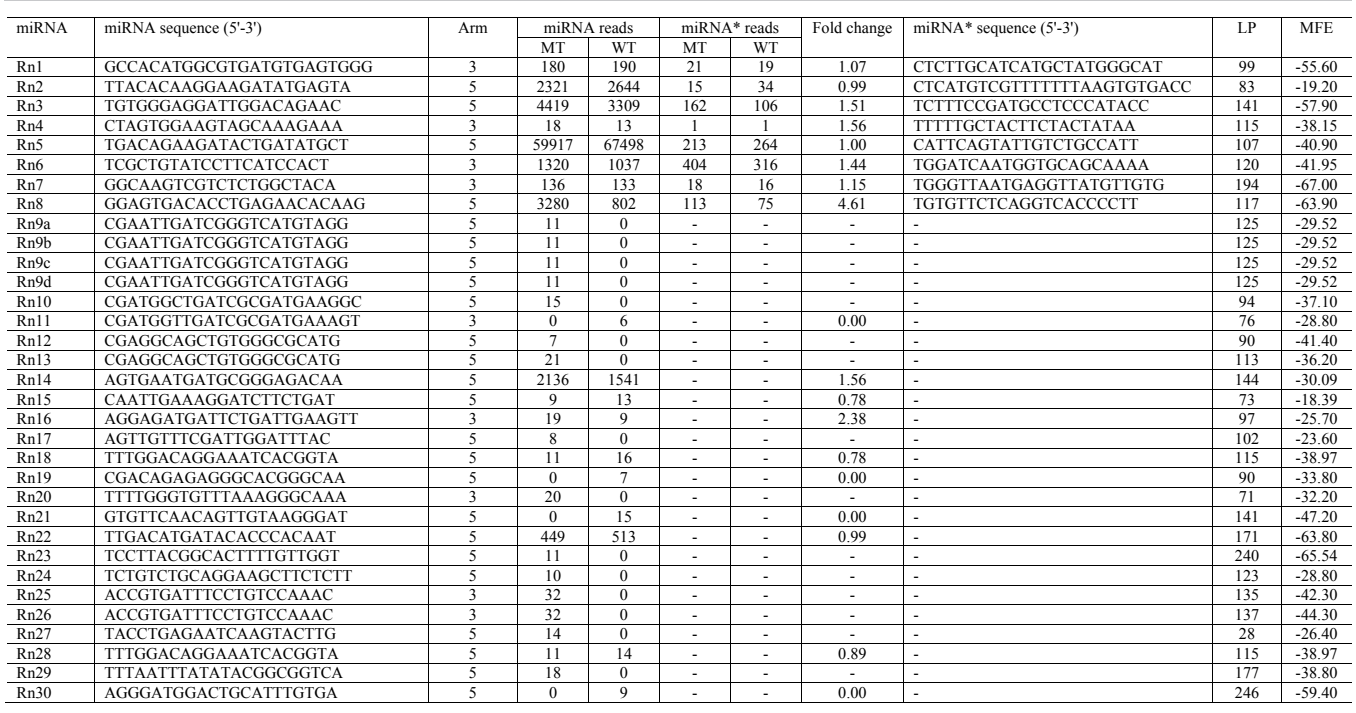

LP (nt): length of precursor; MFE (kcal/mol): minimal folding free energy. Rn1, Rn2, Rn3, Rn4, Rn5, Rn6, Rn7, and Rn8 were novel miRNAs. The rest miRNAs were potential miRNAs.

Table 3. miRNAs with significantly different expression patterns between WT and MT libraries.

\begin{tabular}{|c|c|c|c|c|c|c|}
\hline \multirow[t]{2}{*}{ Members } & \multirow[t]{2}{*}{ miRNA sequence $\left(5^{\prime}-3^{\prime}\right)$} & \multicolumn{2}{|c|}{ miRNA normalized reads } & \multirow[t]{2}{*}{ Fold-change } & \multirow[t]{2}{*}{ Chi-square } & \multirow[t]{2}{*}{ Mode $^{\mathrm{a}}$} \\
\hline & & MT & WT & & & \\
\hline miR160b & TGCCTGGCTCCCTGTATGCCG & 102 & 361 & 0.32 & 0.00 & Down-regulated \\
\hline miR160c & TGCCTGGCTCCCTGTATGCCG & 102 & 361 & 0.32 & 0.00 & Down-regulated \\
\hline miR162 & TCGATAAACCTCTGCATCCAG & 1,363 & 1,000 & 1.54 & 0.00 & Up-regulated \\
\hline miR166a & TCGGACCAGGCTTCATTCCCC & 207,211 & 151,275 & 1.55 & 0.00 & Up-regulated \\
\hline miR166c & TCGGACCAGGCTTCATTCCCG & 125,682 & 89,702 & 1.58 & 0.00 & Up-regulated \\
\hline miR167 & TGAAGCTGCCAGCATGATCTA & 23,301 & 14,346 & 1.83 & 0.00 & Up-regulated \\
\hline $\operatorname{miR} 319 a$ & TTGGACTGAAGGGAGCTCCCT & 512 & 312 & 1.85 & 0.00 & Up-regulated \\
\hline $\operatorname{miR} 319 b$ & TAGCTGCCGACTCATTCATCCA & 1,096 & 581 & 2.13 & 0.01 & Up-regulated \\
\hline Rn3 & TGTGGGAGGATTGGACAGAAC & 4,419 & 3,309 & 1.51 & 0.00 & Up-regulated \\
\hline Rn4 & CTAGTGGAAGTAGCAAAGAAA & 18 & 13 & 1.56 & 0.00 & Up-regulated \\
\hline Rn8 & GGAGTGACACCTGAGAACACAAG & 3,280 & 802 & 4.61 & 0.00 & Up-regulated \\
\hline Rn14 & AGTGAATGATGCGGGAGACAA & 2,136 & 1,541 & 1.56 & 0.00 & Up-regulated \\
\hline Rn16 & AGGAGATGATTCTGATTGAAGTT & 19 & 9 & 2.38 & 0.02 & Up-regulated \\
\hline
\end{tabular}

${ }^{\mathrm{a} E x p r e s s i o n ~ m o d e ~ i n ~ M T . ~}$

\section{qRT-PCR validation}

To validate the sequencing data, the expression of the miRNAs with significantly altered sequencing counts between the two species, including the six known miRNAs (miR160c, miR319a, miR319b, miR166a, miR166c, and miR167) and one novel miRNA (Rn8), was analyzed using qRT-PCR (Figure 3). Similar abundance profiles were obtained by Solexa sequencing and qRT-PCR analysis, indicating that the miRNA expression data determined by Solexa sequencing were reliable. However, there was a discrepancy between the Solexa reads and qRT-PCR results for Rn8. This could have been due to insufficient coverage provided by 
our sequencing depth to reflect the real distribution of these miRNAs, or due to the differing data normalization protocols between the two methods. The qRT-PCR data were normalized relative to the abundance of $5.8 \mathrm{~S}$ rRNA, whereas the sequencing data were normalized relative to the global abundance of all the miRNAs sequenced by Solexa.

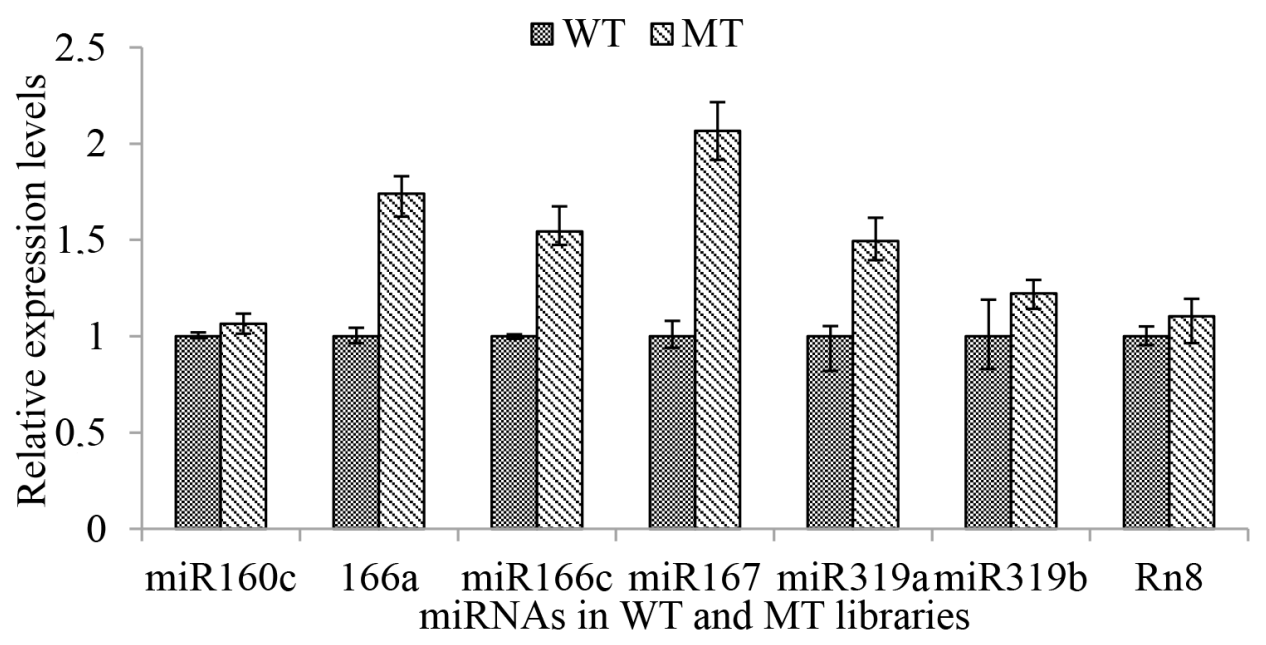

Figure 3. Relative expression levels of miRNAs in WT and MT libraries validated by qRT-PCR. The 5.8S rRNA was used as an endogenous reference gene. The normalized miRNA levels in WT were arbitrarily set at 1.

\section{Target prediction}

Target prediction of six conserved miRNAs and 17 novel and potential miRNAs was performed (Table S4). A total of 47 targets were identified. The number of predicted targets varied from one to four per miRNA. Unfortunately, we were not able to predict the targets of several miRNAs successfully. This could most likely be attributed to a lack of available information on the yellow-horn genome.

The target genes included transcription factors (nuclear transcription factor, class III HD-Zip protein) and genes implicated in disease resistance, antioxidation metabolism (ankyrin repeat-containing protein), nucleobase regulation, and nucleotide and nucleic acid metabolism (alpha DNA polymerase).

\section{DISCUSSION}

miRNAs play crucial roles in flower development and maturation, and have been investigated extensively in several plants. However little is known regarding the molecular mechanisms that regulate petal characters in yellow-horn. We reported miRNAs associated with double flower yellow-horn previously. Due to the lack of nucleotide sequences and ESTs of yellow-horn, the unique small RNAs were aligned against the ESTs of Citrus sinensis 
(Ao et al., 2012) and consequently the results were not very accurate. In the current study, the yellow-horn transcriptome was used to identify unique small RNAs, thus, increasing the reliability of miRNA prediction.

Some novel and conserved miRNAs were found exclusively in the MT or WT library, suggesting that these miRNAs had tissue- or development stage-specific expression. The novel miRNAs displayed lower expression levels compared to the majority of conserved miRNAs. This finding was consistent with the previous studies indicating that non-conserved miRNAs were typically expressed at lower levels.

Differentially expressed genes between the two libraries provided clues to the molecular events of flower development. We also observed the different expression patterns of miRNAs in the present study. Thirteen miRNAs showed significantly different expression between the WT and MT buds. Compared to WT, 11 miRNAs were up-regulated and two were down-regulated in the MT sample, which indicated that they were flower development-related miRNAs.

In this study, miR166, miR167, miR169, and miR319a were significantly differentially expressed between the WT and MT libraries. In previous reports, miR166 over-expression reduced the numbers of pistils and carpels in flowers (Jung and Park, 2007). Notably, miR166a and miR166c read counts were significantly increased (greater than 1.5 -fold change, $\mathrm{P}<0.05$ ) in the MT buds compared to the WT buds. As reported, overexpression of miR166 could cause decrease in the numbers of pistils and carpels. Our finding is consistent with the function of miR166 and suggests that the petaloid pistil and fertility defects in MT buds might be related to over-expression of miR166a and miR166c.

miR167 is critical for defining the correct spatial expression of ARF6 and ARF8 in stamens and ovules to ensure appropriate ovule and anther development for male and female fertility, respectively (Nagpal et al., 2005; Ru et al., 2006; Wu et al., 2006). The MT flowers are sterile, with petaloid stamens and pistil, and exhibit anther dehiscence, and premature arrest of ovule development. miR167 was significantly up-regulated in MT buds, which is in agreement with the fact that its over-expression is associated with sterility.

miR169 controls the fate of floral organ identity during the early stages of flower development by defining the spatial boundaries of the expression of target genes (Cartolano et al., 2007). In our results, miR169a and miR169b expression was increased in MT buds, but not to a statistically significant level.

Another interesting observation involves the identification of miR319 homologs in yellow-horn. miR319a and miR319b were significantly up-regulated in MT buds. miR319a was previously suggested to be a specific regulator of floral organ size and shape. Overexpression of miR319a induces stamen abortion (Palatnik et al., 2007; Efroni et al., 2008; Nag et al., 2009). Consistent with our finding, the stamens of MT buds fuse to small inner petals, and the stigma later becomes petaloid.

In addition to these miRNAs, eight miRNAs (miR160b, miR160c, miR162, Rn3, Rn4, $\mathrm{Rn} 8, \mathrm{Rn} 14$, and Rn16) showed significantly different expressions in MT. The functions of these miRNAs have not been extensively analyzed. They are presumed to be related to flower organ differentiation and development.

Taken together, characterizing the miRNAs expressed in yellow-horn flower buds and understanding the details of the expression patterns of the miRNAs are critical to unraveling their function. Further studies are required to elucidate the regulatory functions of miRNAs in floral development.

Genetics and Molecular Research 15 (4): gmr.15048899 


\title{
Conflicts of interest
}

The authors declare no conflicts of interest.

\section{ACKNOWLEDGMENTS}

\author{
Research supported by the Fundamental Research Funds for the Central Universities \\ (\#2015ZCQ-LX-02).
}

\section{REFERENCES}

Allen RS, Li J, Stahle MI, Dubroué A, et al. (2007). Genetic analysis reveals functional redundancy and the major target genes of the Arabidopsis miR159 family. Proc. Natl. Acad. Sci. USA 104: 16371-16376. http://dx.doi.org/10.1073/ pnas.0707653104

Ao Y (2010). Type classification, individual selection and relevant studies on woody energy plant Xanthoceras sorbifolia Bunge. Chinese Academy of Forestry Press, Beijing.

Ao Y, Wang YW, Chen L, Wang T, et al. (2012). Identification and comparative profiling of microRNAs in wild-type Xanthoceras sorbifolia and its double flower mutant. Genes Genomics 34: 561-568. http://dx.doi.org/10.1007/ $\underline{\text { s13258-012-0065-1 }}$

Cartolano M, Castillo R, Efremova N, Kuckenberg M, et al. (2007). A conserved microRNA module exerts homeotic control over Petunia hybrida and Antirrhinum majus floral organ identity. Nat. Genet. 39: 901-905. http://dx.doi. org/10.1038/ng2056

Efroni I, Blum E, Goldshmidt A and Eshed Y (2008). A protracted and dynamic maturation schedule underlies Arabidopsis leaf development. Plant Cell 20: 2293-2306. http://dx.doi.org/10.1105/tpc.107.057521

Freitas RG, Missio RF, Matos FS, Resende MD, et al. (2011). Genetic evaluation of Jatropha curcas: an important oilseed for biodiesel production. Genet. Mol. Res. 10: 1490-1498. http://dx.doi.org/10.4238/vol10-3gmr1146

Griffiths-Jones S, Saini HK, van Dongen S and Enright AJ (2008). miRBase: tools for microRNA genomics. Nucleic Acids Res. 36: D154-D158. http://dx.doi.org/10.1093/nar/gkm952

Guo HH, Li QQ, Wang TT, Hu Q, et al. (2014). XsFAD2 gene encodes the enzyme responsible for the high linoleic acid content in oil accumulated in Xanthoceras sorbifolia seeds. J. Sci. Food Agric. 94: 482-488. http://dx.doi. org/10.1002/jsfa.6273

Jung JH and Park CM (2007). MIR166/165 genes exhibit dynamic expression patterns in regulating shoot apical meristem and floral development in Arabidopsis. Planta 225: 1327-1338. http://dx.doi.org/10.1007/s00425-006-0439-1

Li R, Li Y, Kristiansen K and Wang J (2008). SOAP: short oligonucleotide alignment program. Bioinformatics 24: 713 714. http://dx.doi.org/10.1093/bioinformatics/btn025

Meyers BC, Axtell MJ, Bartel B, Bartel DP, et al. (2008). Criteria for annotation of plant MicroRNAs. Plant Cell 20: $3186-$ 3190. http://dx.doi.org/10.1105/tpc. 108.064311

Nag A, King S and Jack T (2009). miR319a targeting of TCP4 is critical for petal growth and development in Arabidopsis. Proc. Natl. Acad. Sci. USA 106: 22534-22539. http://dx.doi.org/10.1073/pnas.0908718106

Nagpal P, Ellis CM, Weber H, Ploense SE, et al. (2005). Auxin response factors ARF6 and ARF8 promote jasmonic acid production and flower maturation. Development 132: 4107-4118. http://dx.doi.org/10.1242/dev.01955

Palatnik JF, Wollmann H, Schommer C, Schwab R, et al. (2007). Sequence and expression differences underlie functional specialization of Arabidopsis microRNAs miR159 and miR319. Dev. Cell 13: 115-125. http://dx.doi.org/10.1016/j. devcel.2007.04.012

Ru P, Xu L, Ma H and Huang H (2006). Plant fertility defects induced by the enhanced expression of microRNA167. Cell Res. 16: 457-465. http://dx.doi.org/10.1038/sj.cr.7310057

Schwab R, Palatnik JF, Riester M, Schommer C, et al. (2005). Specific effects of microRNAs on the plant transcriptome. Dev. Cell 8: 517-527. http://dx.doi.org/10.1016/j.devcel.2005.01.018

Shao HB and Chu LY (2008). Resource evaluation of typical energy plants and possible functional zone planning in China. Biomass Bioenergy 32: 283-288.

Sunkar R and Zhu JK (2004). Novel and stress-regulated microRNAs and other small RNAs from Arabidopsis. Plant Cell 16: 2001-2019. http://dx.doi.org/10.1105/tpc.104.022830

Van Gerpen J (2005). Biodiesel processing and production. Fuel Process. Technol. 86: 1097-1107. http://dx.doi.

Genetics and Molecular Research 15 (4): gmr.15048899 
org/10.1016/j.fuproc.2004.11.005

Wang YG, An M, Zhou SF, She YH, et al. (2014). Expression profile of maize microRNAs corresponding to their target genes under drought stress. Biochem. Genet. 52: 474-493. http://dx.doi.org/10.1007/s10528-014-9661-x

Wu MF, Tian Q and Reed JW (2006). Arabidopsis microRNA167 controls patterns of ARF6 and ARF8 expression, and regulates both female and male reproduction. Development 133: 4211-4218. http://dx.doi.org/10.1242/dev.02602

Yao ZY, Qi JH and Yin LM (2013). Biodiesel production from Xanthoceras sorbifolia in China: opportunities and challenges. Renew. Sustain. Energy Rev. 24: 57-65. http://dx.doi.org/10.1016/j.rser.2013.03.047

Zhang JF, Chen GC, Sun QX, Li ZB, et al. (2012). Forest biomass resources and utilization in China. Afr. J. Biotechnol. 11: 9302-9307.

Zhang S, Zu YG, Fu YJ, Luo M, et al. (2010). Rapid microwave-assisted transesterification of yellow horn oil to biodiesel using a heteropolyacid solid catalyst. Bioresour. Technol. 101: 931-936. http://dx.doi.org/10.1016/j. biortech.2009.08.069

\section{Supplementary material}

Figure S1. Secondary structures of conserved miRNAs precursors in yellow-horn.

Figure S2. Secondary structures of yellow-horn-specific miRNAs precursors.

Table S1. List of primer sequences used for qRT-PCR.

Table S2. Small RNA annotations in WT and MT libraries.

Table S3. Conserved miRNAs in WT and MT libraries.

Table S4. Target genes of novel and conserved $X$. sorbifolia miRNAs. 